\title{
A Study of Integration of a Rotating Detonation Engine to a Waverider Forebody
}

\author{
F.K. Lu, A.R. Mizener \\ Aerodynamics Research Center, Mechanical and Aerospace Engineering Department, \\ University of Texas at Arlington, Arlington, Texas 76019, USA \\ P.E. Rodi \\ Mechanical Engineering Department, William Marsh Rice University, Houston, Texas \\ 77005, USA
}

Corresponding Author's name: franklu@uta.edu

\begin{abstract}
An ideal parametric analysis was performed by combining a waverider forebody generated by the osculating flowfield method and a second-order performance model for a rotating detonation engine. A sharp nose and a slightly convex forebody profile yielded the greatest pressure recovery and greatest installed engine performance. Engine performance improved with increasing Mach number, but propellant autoignition temperature limits may limit Mach numbers to less than 3.5.
\end{abstract}

\section{Introduction}

The promise of detonations for aerospace propulsion has prompted attempts to realize such engine concepts for at least sixty years [1]. These attempts can be conveniently classified into a few main detonation engine genres as shown schematically in Fig. 1. The upper left shows an oblique detonation wave engine (ODWE) [2]. The incoming flow of premixed propellant exceeds the Chapman-Jouguet (CJ) speed which results in a steady oblique detonation wave forming over a sharp wedge indicated by the dotted line in the figure. To the right is a rotating detonation engine (RDE) which is generally considered steady although it is strictly not so $[3,4]$. The curved arrow in the figure indicates a detonation wave traveling circumferentially in an annular chamber exhausting burnt products to the right. Due to the high frequency of operation of $\mathcal{O}(1-10) \mathrm{kHz}$, for application purposes it is considered steady although care must be exercised in interpreting the cyclic data. Below the RDE is a depiction of a pulsed detonation engine (PDE) where repeated, periodic ignition of the propellants causes detonation waves to propagate unidirectionally to the exit of the detonation tube $[5,6]$. Design studies have indicated that a PDE must operate in the 50-200 Hz range for aerospace applications [7]. Finally, the lower left depicts linear detonation engine (LDE) which is a configuration that has not been well studied. It is the linear equivalent of the RDE in that detonation waves travel back-and-forth in a detonation chamber instead of circumferentially around one. This type of normal detonation wave process had been considered to be unstable and thus unsuitable for propulsion applications [2]. However, Wilson et al. [8] found that it is possible to allow the detonation wave to undergo a stable oscillatory motion if the incoming flow is at a sub-CJ Mach number. Depending on the length of the chamber, an LDE can operate at frequencies comparable to an RDE. There may be some further advantages in the LDE compared to the RDE in terms of scalability and ease of fueling but apparently not much research has been paid to this concept.

The most commonly cited reason for pursuing detonation engines is the reduced entropy rise for the same ideal work produced as discussed in standard texts on combustion 

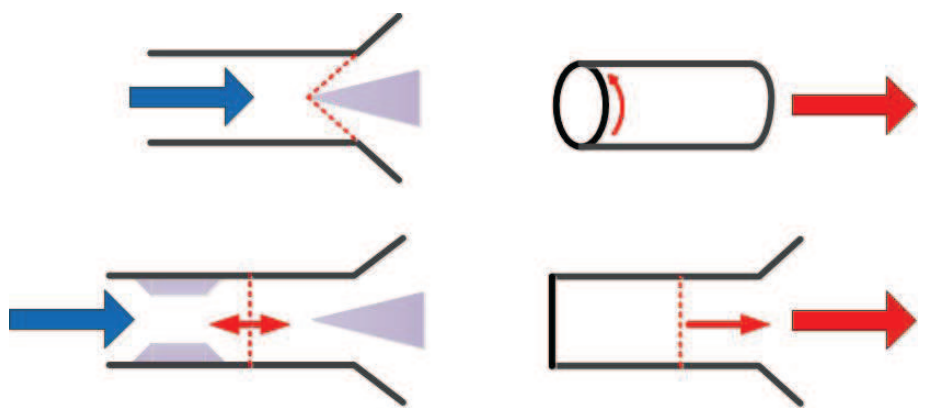

Fig. 1 Taxonomy. Clockwise from upper left: oblique detonation wave engine, rotating detonation engine, pulsed detonation engine, "linear" detonation engine

and detonation; this reduced entropy rise translates to improved thermodynamic efficiency. In the late 1990's, attention was heavily focused on PDEs and an understanding of their thermodynamic and propulsive efficiencies arose [9]. Less developed are similar analytical tools for RDEs and only recently have some efforts been undertaken $[10,11,12]$.

It is necessary to further understand the improvement in theoretical thermodynamic efficiency in terms of actual operational conditions. One such approach is to integrate an engine to an aircraft for a given mission profile, thereby providing other metrics such as range in addition to the usual performance metrics such as specific impulse or specific thrust. Moreover, a comparison between a detonation-based engine and conventional technologies such as a Brayton engine does not appear to be available in the literature. Despite this comment, an actual vehicle utilizing a PDE [13] had flown and demonstrators for both PDE and RDE had also been developed $[14,15]$. Moreover, this paper does not directly address a comparison between an RDE-powered vehicle against a conventional Brayton-powered one and much work remains in this respect. Instead, this paper reports a recent study aimed at developing a reduced-order model for an RDE and its integration to the forebody of a hypersonic vehicle [16].

\section{Waverider Forebody}

Studies aimed at integrating an airframe to an RDE are practically nonexistent due to the novelty of the engine concept. Certain concepts have been proposed or investigations of partial component integration have been reported [17, 18, 19, 20, 21, 22, 23]. As a preliminary step toward a complete airframe/engine integration and trajectory analysis, a study of an RDE integrated to a waverider forebody was presented [16], based on the analysis in [12]. Exernal forebody compression is a crucial aspect in the design of a high-speed airbreathing vehicle. The tight airframe/engine integration requires that the forebody contribute optimally to the lift-to-drag $(L / D)$ ratio as can be provided by a waverider. For an integrated airbreather, the forebody must efficiently compress the flow, provide adequate mass capture with minimum inlet flow distortion, spillage and separation and be matched optimally with the inlet over a wide Mach number range. Moreover, the forebody affects the entire vehicle configuration through sensitivities to the incidence and sideslip and so forth [24].

Rodi $[25,26]$ found that osculating flowfield-based waveriders (OFWRs) are particularly suitable for an integrated airbreather with improved lift-to-drag ratio. The osculating 
flowfield method uses planes that are normal to the local shock wave shape that are defined starting at the trailing edge of the waverider geometry. Flowfields from geometries that are beyond right angle cones are permitted on each osculating plane. This approach is an improvement of a previous osculating cone method [27, 28].

Mizener et al. [16] conducted a limited study on integrating an RDE to the forebody of an OFWR. In order to do this, a modification was made to a previous, reduced-order model for a RDE in rocket mode [12]. A low-order model allows for parametric sweeps to be conducted rapidly with little computational expense. These features are desirable for early, design-stage analysis and engine sizing where detailed modeling of the flow may not be warranted.

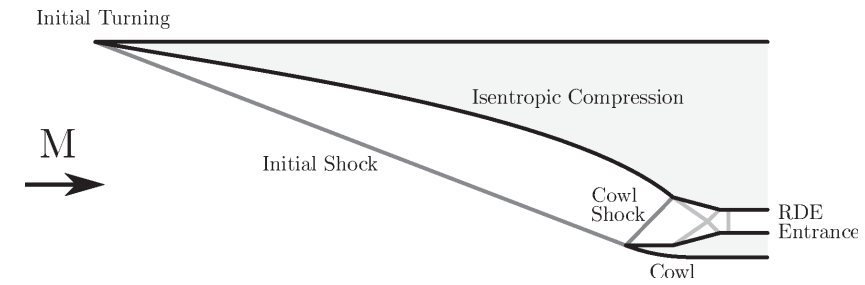

Figure 2: Assumed forebody flowfield [26]

\subsection{OFWR Forebody}

Define a power-law body by

$$
\frac{r}{r_{b}}=\left(\frac{x}{L}\right)^{n}
$$

where $r$ is the local radius, $r_{b}$ is the radius of the at its base, $x$ is the vehicle axis measured from the nose, $L$ is the total forebody length, and $n$ is the exponent in the power-law body expression, typically $0.75-1.1$ such that an exactly conical geometry is produced when $n=1$. The cone angle $\theta_{\text {cone }}$ can vary across the span of the vehicle, using the effective shock wave angle $\beta_{\text {eff }}$ to define the streamwise distance between the leading edge and the traces in the waverider baseplane. Equation (1) yields the local surface turning angle at any location on a given osculating plane

$$
\theta_{s}=\tan ^{-1}\left[n \sin \theta_{\text {cone }}\left(\frac{x}{L}\right)^{n-1}\right]
$$

Equation (2) defines the initial turning angle of the flow at the leading edge and the final turning at the rear of the osculating plane (that is, just before the cowl shock), see Fig. 2. For the initial turning angle at the leading edge, $x / L$ is set to a small value and Eq. (2) is used to generate the local turning. For non-conical bodies $(n \neq 1), x / L \approx 0$ to ensure small initial turning values. Note that a small initial turn is desirable for off-design considerations. For a physically realistic example, a 1 in $(25.4 \mathrm{~mm})$ radius leading edge on a $100 \mathrm{ft}(30.5 \mathrm{~m})$ long osculating plane yields $x / L=8.33 \cdot 10^{-4}$. For this value of $x / L$, and with $\theta_{\text {cone }}=11 \mathrm{deg}$ and $n=1.1$, the initial flow turning angle $\theta_{s}=5.9 \mathrm{deg}$. The oblique shock due to this initial turning angle, equivalent to a two-dimensional wedge, will set the conditions of the flow entering the engine. Local air properties just downstream of the leading edge are calculated using the initial turning angle for the given osculating plane using the Rankine-Hugoniot relations. 
Other than the initial, non-isentropic turn, the flow downstream of the leading edge is turned isentropically to the turning angle at the downstream end of the osculating plane, that is, immediately before the engine. At $x / L=1$ for this particular geometry, the final flow turning angle is $\theta_{s}=11.85 \mathrm{deg}$. This flow is then turned back to the freestream direction by an oblique shock wave, resulting in the conditions entering the engine, see Fig. 2. Note that the figure includes features that will be described later. Any flowfield non-uniformities normal to the forebody surface are ignored.

\subsection{Flight Conditions}

The airbreathing corridor is typically regarded broadly to be where the dynamic pressure $\bar{q}=500-2000 \mathrm{psf}(24-95 \mathrm{kPa})$. It is convenient to display the dynamic pressure in an altitude versus speed or Mach number plot upon which can also be plotted the trajectories of aerospace vehicles. It is obvious that the airbreathing corridor represents a balance between aerodynamic, structural and propulsion considerations. In particular consideration for RDE operation (or any detonation engine) is that the propellants must not auto-ignite prior to the detonation wave. Dynamic pressures of 500, 1000 and 1500 psf $(24,48,72 \mathrm{kPa})$ are considered in the present study. A full consideration of auto-ignition characteristics is beyond the scope of this paper [29, 30, 31]. Here, autoignition temperature is taken to be the stagnation temperature of the flow at the engine inlet $T_{0_{i n j}}$ which is a function of the freestream Mach number $M_{\infty}$ and the dynamic pressure $\bar{q}$. Mizener et al. [16] summarized the data for hydrogen and selected hydrocarbons as reproduced in Table 1. The autoignition temperatures (AITs) are only very weakly dependent on pressure, being more dependent on the equivalence ratio. Taking the worst case of the lower AIT, the maximum freestream Mach number that can be achieved for the three dynamic pressures considered are also displayed in Table 1. The data presented in Table 1 indicate that $M_{\infty} \lesssim 3.7$ based on the lower AIT value for most fuels, being much lower for JP-8. In this paper, JP-8 will not be considered and subsequent design will focus on $M_{\infty}=3$ and 3.5 as example freestream Mach numbers for the three dynamic pressure conditions.

Table 1 Maximum freestream Mach number at lower autoignition limit of selected fuels

\begin{tabular}{cccccc}
\hline \multirow{2}{*}{ Fuel } & \multirow{2}{*}{ Formula } & AIT & \multicolumn{3}{c}{ Maximum freestream Mach No. $M_{\infty}$} \\
\cline { 4 - 6 } & & Range $(\mathrm{K})$ & $\bar{q}=500 \mathrm{psf}$ & $1000 \mathrm{psf}$ & $1500 \mathrm{psf}$ \\
\hline Hydrogen & $\mathrm{H}_{2}$ & $773-793$ & 3.53 & 3.58 & 3.58 \\
Methane & $\mathrm{CH}_{4}$ & $810-905$ & 3.65 & 3.69 & 3.70 \\
Propane & $\mathrm{C}_{3} \mathrm{H}_{8}$ & $739-766$ & 3.43 & 3.47 & 3.47 \\
Ethylene & $\mathrm{C}_{2} \mathrm{H}_{4}$ & $723-763$ & 3.34 & 3.38 & 3.38 \\
Methanol & $\mathrm{CH}_{3} \mathrm{OH}$ & $698-715$ & 3.29 & 3.33 & 3.33 \\
JP-8 & & $500-550$ & 2.55 & 2.56 & 2.56 \\
\hline
\end{tabular}

\subsection{Forebody Selection}

Mizener et al.'s [16] study of integration of the waverider forebody with the inlet found that the configuration depicted in Fig. 2 with a crossing shock system and a terminal 
normal shock in the internal inlet achieves good pressure recovery. Contour plots of the total pressure ratio of the inlet behind the final normal shock, normalized by the freestream total pressure, are shown in Figs. 3 and 4 for $M_{\infty}=3$ and 3.5. The forebody cone angle varied from 5-8 deg for $M_{\infty}=3$ or 5-9 deg for $M_{\infty}=3.5$, while the $n$-factor varied from $0.75-1.1$. Solutions were obtained only for attached shock solutions within the inlet. Shock detachment due to low values of $n$ were excluded and shown as gray regions in the figures.

At Mach 3, the best performing forebodies have a total pressure ratio of approximately 0.88 , found along a region $\left\{\theta_{\text {cone }}, n\right\}=\{5 \mathrm{deg}, 0.86\} \rightarrow\{8 \mathrm{deg}, 0.95\}$. At Mach 3.5 , the best performing forebodies have a lower total pressure ratio of approximately 0.78 , found along a region $\left\{\theta_{\text {cone }}, n\right\}=\{5 \mathrm{deg}, 0.84\} \rightarrow\{9 \mathrm{deg}, 0.94\}$, comparable to values at $M_{\infty}=3$. These results are consistent with [32].

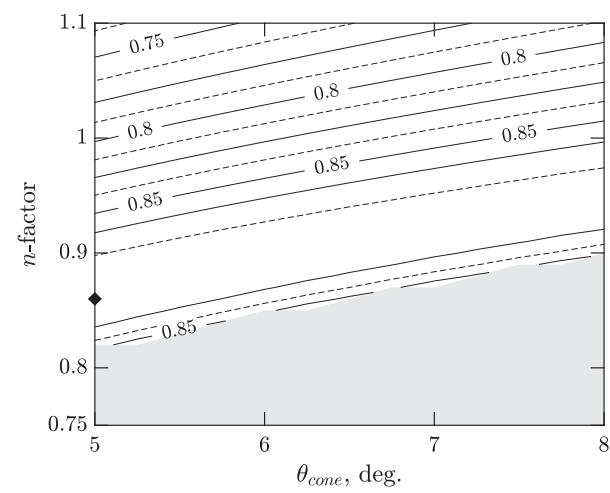

Figure 3: Total pressure ratio for $M_{\infty}=3$.

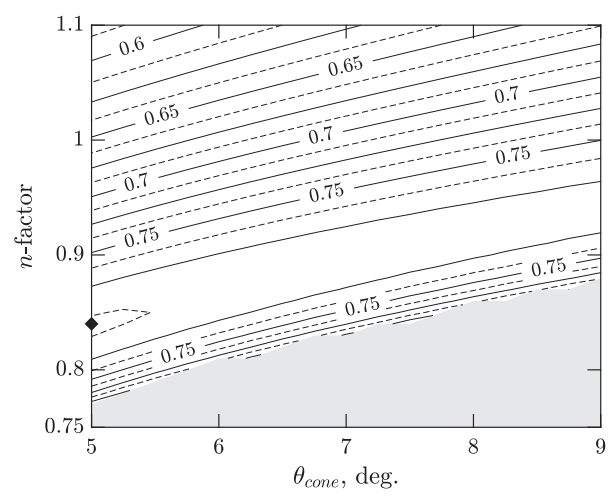

Figure 4: Total pressure ratio for $M_{\infty}=$ 3.5 .

\section{Rotating Detonation Engine Modeling}

A straightforward forebody/engine integration is proposed where the flowfield exiting the forebody model defines the inflow conditions for the RDE model based on a rotating detonation rocket engine [12]. A semi-empirical, control volume was used and only a brief description is provided here. At the RDE entrance, a uniform stoichiometric mixture of fuel and air is assumed. Flow within the RDE is assumed to be cyclic and inviscid with no body forces. The engine is modeled as an annulus with no cross-sectional area change or nozzle. Property variations in the radial direction are expected to be small and are thus neglected. Fuel-air mixing and the effect of contact surface burning are neglected. Only a single detonation wave is assumed.

Design parameters for the engine consist of the annulus outer and inner diameters $d_{o}$ and $d_{i}$ and circumference $L$, the fuel-air mixture plenum stagnation pressure and temperature $P_{0_{i n j}}$ and $T_{0_{i n j}}$, the fuel-air mixture and the equivalence ratio $\phi$. Detonation properties are calculated using Cantera [33] with the Caltech Shock \& Detonation Toolbox [34], assuming choked injector flow upstream of the detonation wave and equilibrium conditions, utilizing the USC-II mechanism [35].

Figure 5 shows an unrolled view of the RDE internal flowfield whereby a detonation wave is propagating circumferentially around the annuluar chamber sustained by con- 


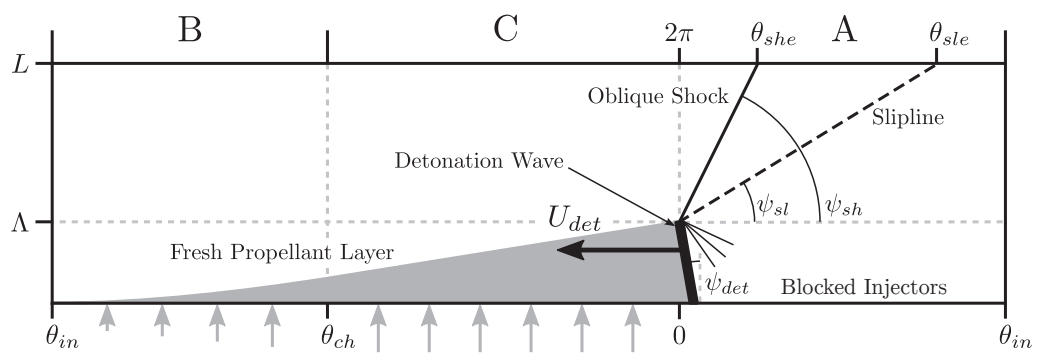

Figure 5: Schematic of RDE internal flowfield.

tinual axial propellant injection. The combustion products expand upward, out of the rear of the engine to produce thrust. The hot combustion products expanding behind the detonation wave interact with recirculating products from the previous passage of the detonation wave, resulting in an oblique shock wave and a slipline between the fresh detonation products, and those recirculating and passing through the shock wave. Numerical simulations have shown that both the oblique shock wave and slipline curve slightly around the annulus. However, as a first-order approximation, the oblique shock and the slipline are considered to be straight [12].

The flow entering the RDE is considered to be subsonic. One period of the cyclic detonation wave passage can be spatially divided into three flow regimes (Fig. 5):

A $P_{c} \geq P_{0_{i n j}}:$ No injection. Counter-flow is neglected in this model.

B $P_{0_{i n j}}>P_{c}>P_{c r}$ : Flow entering the chamber is subsonic, $P_{i n j}=P_{c}$.

C $P_{c} \leq P_{c r}$ : Flow entering the chamber is sonic, $P_{i n j}=P_{c r}$.

where $P_{c}$ is the chamber static pressure, $P_{i n j}$ is the static pressure of the injected propellant flow, and $P_{c r}$ is the critical pressure. The injection temperature and velocity in $\mathrm{B}$ and $\mathrm{C}$ are dictated by the injection pressure ratio $P_{i n j} / P_{0_{i n j}}$. The points at which injection begins (separating A from B) and chokes (separating B from C) are designated $\theta_{\text {in }}$ and $\theta_{c h}$, respectively. This models flow into the chamber immediately upstream of the detonation wave as sonic, resulting in a lower static pressure and therefore a lower detonation pressure. Since RDE performance is strongly dependent on the detonation pressure, this model results in increased predicted performance over a subsonic injector model. Further research into RDE integration with airbreathing forebodies, particularly for supersonic applications, will require a more detailed investigation of inlet/diffuser design and behavior.

The control volume analysis is performed in a noninertial, detonation-fixed coordinate system and reverted back to the inertial reference frame for computing performance parameters. This Galilean transformation was previously employed for RDEs by Nordeen et al. [36], namely,

$$
\vec{W}=\vec{V}-\vec{U}
$$

where $\vec{W}$ is the velocity in the noninertial reference frame, $\vec{V}$ is the velocity in the inertial reference frame and $\vec{U}$ is the velocity of the noninertial frame with respect to the inertial frame. For an RDE, $\vec{U}$ is the detonation wave velocity.

Numerical models have indicated that the detonation wave inclines somewhat towards the injector flow. The detonation wave inclination angle can be obtained by assuming 
the detonation wave is normal to the noninertial velocity vector of the injection flow immediately upstream of the detonation wave yielding

$$
\psi_{\text {det }}=\tan ^{-1} \frac{W_{\mathrm{I}_{z}}}{W_{\mathrm{I}_{\theta}}}
$$

where $\vec{W}_{\mathrm{I}}$ is the noninertial velocity vector immediately upstream of the detonation wave.

The oblique shock and slipline angles $\psi_{s h}$ and $\psi_{s l}$ are calculated by the method of Sichel and Foster [37]. The inclination of the detonation wave has the effect of increasing the shock angle and sliplines with respect to the inlet face. To account for this, the shock and slipline angles computed by the method given here are respectively adjusted:

$$
\begin{aligned}
& \psi_{s h}=\psi_{s h}+\psi_{\text {det }} \\
& \psi_{s l}=\psi_{s l}+0.5 \psi_{\text {det }}
\end{aligned}
$$

Figure 5 shows that the detonation products expand in a region bounded above by the slipline and below by the injector face and fresh propellant layer. This expansion flow is similar to that seen in an aerospike nozzle. With the detonation wave forming a pseudo-throat and the area change a known function of $\theta$, the expansion region can be computed readily using the Mach-area relationat any point in the expansion, with the sonic area taken as the detonation wave surface area. The expansion area is a function of the detonation wave height $\Lambda$. This is dictated by the pressure decay inside the annulus through a numerical solution [16].

A portion of the flow expansion in an RDE recirculates and passes through the oblique shock wave $[38,39]$. This causes flow reversal in the inertial frame, counteracting the detonation-induced circumferential velocity component. This flow turning is determined through approximating the variation of the flow angle on the exit plane. In the noninertial frame, the slipline exits the annulus at an angle $\psi=\psi_{s l}$. Therefore, the angle at which the flow impinges upon the shock wave where it exits the annulus can be approximated by a streamline beginning at the base of the detonation wave. This turn can be determined from oblique shock equations, which then sets the flow angle along the entire exit plane.

Flow properties are needed along the exit plane and are determined by applying the Mach-area relation to first determine the Mach number in the noninertial frame $M_{w}$. For $\theta_{\text {sle }} \leq \theta \leq 2 \pi$, the area is assumed to be bounded above by the slipline and below by the injection plane $\left(0 \leq \theta \leq \theta_{i n}\right)$ or injected propellant layer $\left(\theta_{i n} \leq \theta \leq 2 \pi\right)$. For flow expansion past $\theta=2 \pi$, an extension of this method is required. For $\theta=\left[2 \pi, \theta_{\text {sle }}+2 \pi\right]$, the lower bound of the expansion area is approximated by the mean of the shock and slipline angles, and for $\theta=\left[\theta_{\text {she }}+2 \pi, \theta_{\text {sle }}+2 \pi\right]$ the upper and lower bounds are the shock wave and slipline, respectively. Once the expansion area is determined, the noninertial Mach number is then used to obtain the magnitude of the inertial velocity $\|\vec{W}\|=M_{w} a$. A flow-angle constraint is applied to this to determine the $z$-and $\theta$-components $W_{z}$ and

$W_{\theta}$. Finally, once the components of $\vec{W}_{e}$ are known, the exit velocity in the inertial frame $\vec{V}_{e}$ is calculated by the Galilean transformation, Eq. (3).

\section{Performance Model}

The control volume used for this analysis is shown in Fig. 6. The inlet is defined as surface 1 and consists of uniform flow at freestream conditions. The exit, surface 2, is defined as the RDE exit plane, and properties are determined by the RDE analysis given 


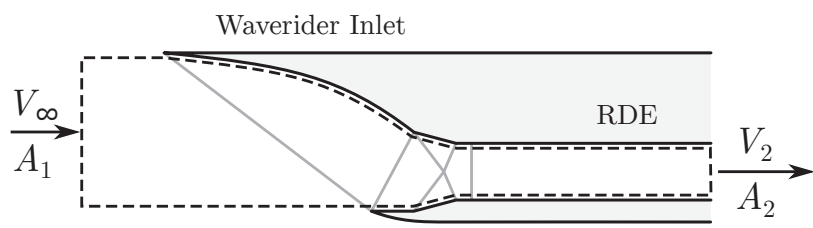

Figure 6: Integrated RDE/waverider control volume

above. Further surfaces are drawn such as to follow streamlines. Details of inlet design are beyond the scope of the current analysis.

Expressions for the thrust and circumferential force are formulated by performing a momentum balance on the control volume, namely,

$$
\begin{aligned}
& F_{z}=\delta_{a} r_{m} \int_{0}^{2 \pi}\left(\rho V_{z}^{2}+P-P_{\infty}\right)_{2} d \theta-\rho_{\infty} V_{\infty}^{2} A_{1} \\
& F_{\theta}=\delta_{a} r_{m} \int_{0}^{2 \pi}\left(\rho V_{\theta} V_{z}\right)_{2} d \theta
\end{aligned}
$$

where the ambient back pressure is calculated from the freestream Mach number $M_{\infty}$, the inlet pressure ratio $P_{0_{i n j}} / P_{0_{\infty}}$, and the RDE inlet stagnation pressure, assuming a ratio of specific heats of $\gamma=1.4$,

$$
P_{\infty}=P_{0_{i n j}}\left(\frac{P_{0_{i n j}}}{P_{0_{\infty}}}\right)^{-1}\left(1+\frac{\gamma-1}{2} M_{\infty}^{2}\right)^{-\gamma /(\gamma-1)}
$$

and the inlet area

$$
A_{1}=\frac{1}{\rho_{\infty} V_{\infty}} \int_{2}(\rho V)_{2} d A_{2}
$$

In addition to thrust and circumferential force, other performance parameters include the torque calculated about the mean annular radius

$$
\mathcal{T}=r_{m} F_{z}
$$

the fuel-based specific impulse

$$
I_{s p}=\frac{F_{z}}{\dot{m}_{f} g_{0}}
$$

and the thrust-specific fuel consumption

$$
\mathrm{TSFC}=\frac{\dot{m}_{f}}{F_{z}}
$$

For a further discussion of circumferential force and torque in an RDE capacity, see [12].

\section{$5 \quad$ RDE Sizing}

The Mach 3-4 region is of interest for air-breathing cruise missiles and appears suitable for implementing RDEs. While waveriders are typically considered for hypersonic cruise, they were proposed at the lower Mach number region [40]. Amongst medium-to-long range cruise missiles in the Mach 2-5 range, the propulsion system is either a ramjet or 
a gas turbine, with mention that there is at least one effort to produce an RDE-powered cruise missile [4].

A critical consideration on the minimum size of a detonation engine are the minimum dimensions to ensure a successful propagation of the detonation wave. Detonation waves are known to fail to propagate in narrow channels, and a limit for RDE operation as a function of detonation cell size $\lambda$ was proposed by Bykovskii [41] as

$$
\delta_{a_{\text {min }}} \gtrsim(2.4 \pm 1) \lambda
$$

where $\delta_{a_{\min }}$ is the minimum annulus width. Examples of fuels with relatively small detonation cell size for stoichiometric combustion in air at or near standard temperature and pressure are shown in Table 2. The present study considers only hydrogen and propane. Hydrogen has a small cell size and has a high heating value although it is difficult to store and is volumetrically inefficient. Propane was selected as a surrogate for liquid hydrocarbon blends. It is easily storable and offers a moderately high autoignition temperature.

Table 2: Stoichiometric detonation cell sizes for selected fuels in air at standard conditions

\begin{tabular}{|c|c|c|c|}
\hline \multirow[b]{2}{*}{ Name } & \multicolumn{3}{|c|}{ Detonation Cell } \\
\hline & Formula & Size $\lambda(\mathrm{mm})$ & Reference \\
\hline Hydrogen & $\mathrm{H}_{2}$ & 15.1 & {$[42]$} \\
\hline Methane & $\mathrm{CH}_{4}$ & $280-320$ & {$[42]$} \\
\hline Propane & $\mathrm{C}_{3} \mathrm{H}_{8}$ & 69 & {$[42]$} \\
\hline Ethylene & $\mathrm{C}_{2} \mathrm{H}_{4}$ & $21.2-28$ & {$[42,43]$} \\
\hline Methanol & $\mathrm{CH}_{3} \mathrm{OH}$ & 26 & {$[44]$} \\
\hline JP-4 & & 46.0 & {$[43]$} \\
\hline
\end{tabular}

A survey of small turbojets and turbofans engines used for cruise missiles indicate that they have outer diameters of $d_{o} \approx 0.3 \mathrm{~m}$. Thus an outer diameter of $0.3 \mathrm{~m}$ for the annulus is used for the RDE from which other lengths are determined such as the annulus width $\delta_{a}$ and length $L$. Performance results for hydrogen and propane are shown in Figs. 7 and 8 respectively for inlet conditions from a conical forebody $\left(\theta_{\text {cone }}=5 \mathrm{deg}, n=1.0\right)$ at $M_{\infty}=3, \bar{q}=1000 \mathrm{psf}(48 \mathrm{kPa})$. The results show that wider annuli yield greater thrust but with reduced specific impulse. A longer chamber decreases both thrust and specific impulse, and lowers the resultant torque on the control volume. From these observations, a configuration with $\delta_{a}=50 \mathrm{~mm}, d_{i}=0.2 \mathrm{~m}$ and $L=0.3 \mathrm{~m}$ appear optimum for the above conditions.

\section{Forebody/RDE Integrated Performance}

Additional performance analysis was conducted and the complete list of cases is listed in Table 3. The data are plotted as contour maps of thrust $F_{z}$, torque $\mathcal{T}$, fuel-based specific impulse $I_{s p}$, and thrust-specific fuel consumption TSFC vs. $n$ and $\theta_{\text {cone }}$ in Figs. 9 and 10 for hydrogen and propane respectively. No solutions were presented for shock detachment as would occur for blunt convex body profiles $n \leq 0.9$, especially for larger values of $\theta_{\text {cone }}$. Mach 3 cases are limited to a maximum cone angle of $8 \mathrm{deg}$ and no solutions are possible below $n=0.82$. Mach 3.5 cases are limited to $9 \mathrm{deg}$ and no solutions exist below $n=0.77$. Regions where no solutions exist again shaded gray on the contour plots. For each plot, the point of maximum performance is marked with a black diamond. 


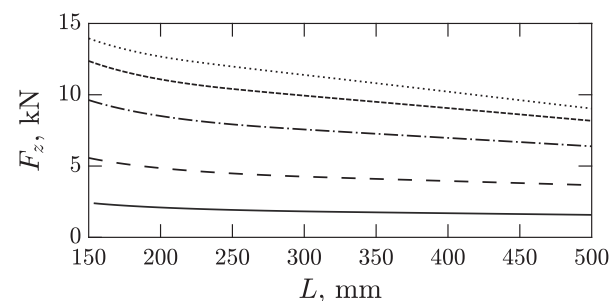

(a) Thrust $F_{z}$

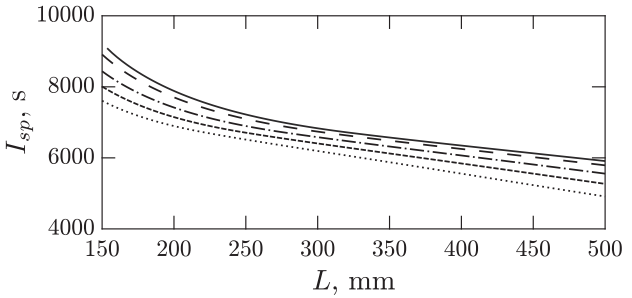

(c) Fuel-based specific impulse $I_{s p}$

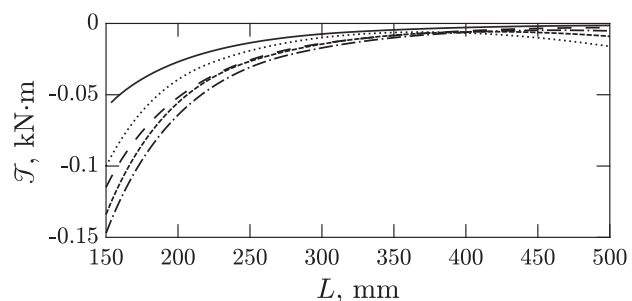

(b) Torque $\mathcal{T}$

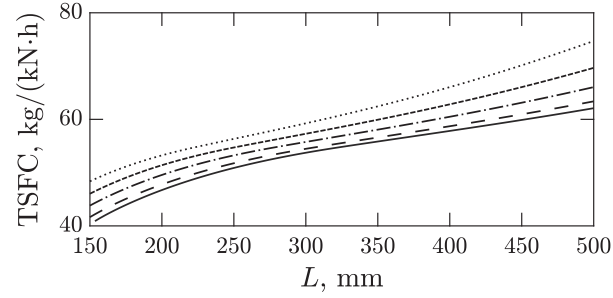

(d) Thrust specific fuel consumption TSFC $\delta_{a}=50 \mathrm{~mm}-\delta_{a}=75 \mathrm{~mm} \quad \cdots \cdots \cdots \cdots \cdots \delta_{a}=100 \mathrm{~mm}$

Figure 7: RDE sizing results, hydrogen

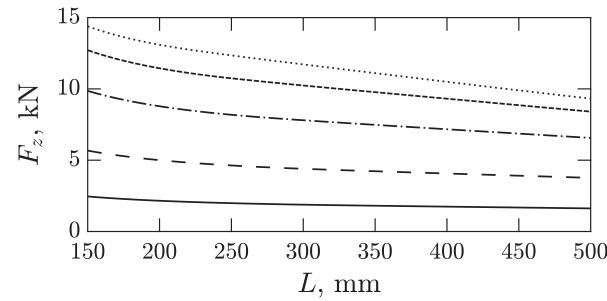

(a) Thrust $F_{z}$

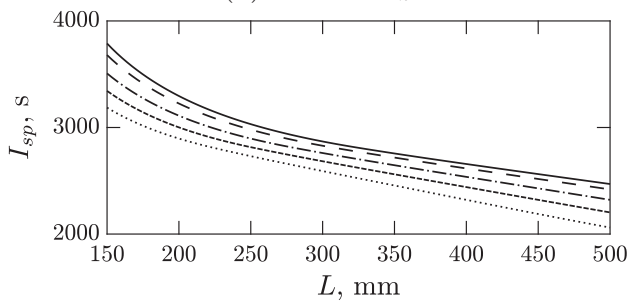

(c) Fuel-based specific impulse $I_{s p}$

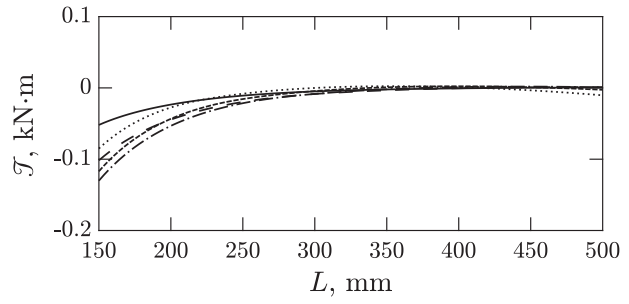

(b) Torque $\mathcal{T}$

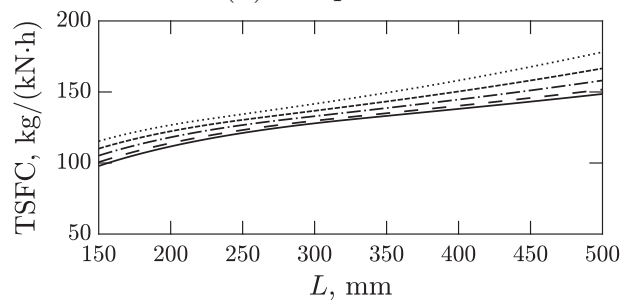

(d) Thrust specific fuel consumption TSFC

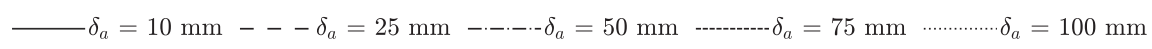

Figure 8: RDE sizing results, propane 
Table 3: Summary of design cases

\begin{tabular}{ccc}
\hline $\begin{array}{c}\text { Mach } \\
\text { No. } M_{\infty}\end{array}$ & $\begin{array}{c}\text { Dynamic } \\
\text { pressure } \bar{q}, \mathrm{psf}(\mathrm{kPa})\end{array}$ & Fuel \\
\hline 3 & $500(24)$ & $\mathrm{H}_{2}$ \\
3 & $1000(48)$ & $\mathrm{H}_{2}$ \\
3 & $1500(72)$ & $\mathrm{H}_{2}$ \\
3.5 & $1000(48)$ & $\mathrm{H}_{2}$ \\
3 & $500(24)$ & $\mathrm{C}_{3} \mathrm{H}_{8}$ \\
3 & $1000(48)$ & $\mathrm{C}_{3} \mathrm{H}_{8}$ \\
3 & $1500(72)$ & $\mathrm{C}_{3} \mathrm{H}_{8}$ \\
3.5 & $1000(48)$ & $\mathrm{C}_{3} \mathrm{H}_{8}$ \\
\hline
\end{tabular}

Figures 9 and 10 give the performance results for $M_{\infty}=3, \bar{q}=1000$ psf, with hydrogen and propane fuel, respectively. The optimum $n$-factor increases to $n=0.95$ at $\theta_{\text {cone }}=8$ deg. Performance is relatively insensitive to cone angle whereby increasing $\theta_{\text {cone }}$ along the performance peak from 5-8 deg results in a decrease in thrust of less than 0.4 percent for both fuels. Propane offers a 3 percent increase in thrust and a 51 percent decrease in torque over hydrogen, but vastly decreases specific impulse (and increases TSFC) by a factor of 2.4. These factors are due to the much higher weight of propane over hydrogen. While at $M_{\infty}=3$, raising the dynamic pressure to $1500 \mathrm{psf}(72 \mathrm{kPa})$ does not change the point of peak performance. But the 50 percent higher dynamic pressure obviously results in a 50 percent increase in thrust. Torque increases proportionally less, by 45.5 percent for hydrogen and 50.3 percent for propane. Specific impulse and TSFC are negligibly affected for either hydrogen or propane.

Finally, increasing the freestream Mach number to $M_{\infty}=3.5$ with $\bar{q}=1000$ psf (48 $\mathrm{kPa}$ ) changes the optimum forebody characteristics with peak performance occurring at $\theta_{\text {cone }}=5 \mathrm{deg}$ and $n=0.84$. Further, while the performance maps have the same general shape, they no longer scale identically. There is an increased performance due to the higher pressure at the RDE inlet. Peak thrust, relative to the maximum performance of the baseline case, increases by 20.3 percent for hydrogen and by 18.8 percent for propane. Torque increases at a proportionally greater rate, particularly for propane: by a factor of 2.04 for hydrogen vs. a factor of 2.73 for propane. Specific impulse and TSFC are not significantly affected; the former decreases by three percent (hydrogen) and four percent (propane), the latter decreases by just over three percent (hydrogen) and just over four percent (propane), respectively.

From the above results, the thrust is found to be sensitive to body shape $\left(\theta_{\text {cone }}, n\right)$ and freestream $\left(M_{\infty}, \bar{q}\right)$ parameters, and scales with the pressure at the RDE inlet. Torque scales similarly with thrust (though proportionally somewhat less in $\bar{q}$, and somewhat more in $M_{\infty}$ ). Fuel-based specific impulse and TSFC follow the same trends as thrust, but are far less sensitive to perturbations in either body shape or freestream parameters. This is because the mass flow rate and thrust scale similarly with changes in $P_{0_{i n j}}$. Greater performance is achieved at higher Mach numbers and dynamic pressures. However, autoignition temperature limits may present a barrier to operation at $M_{\infty}>3.5$. This temperature limit is eased somewhat at higher dynamic pressures since $T_{0_{i n j}}$ decreases with increasing $\bar{q}$, indicating that higher Mach number operation may be easier to accomplish under such circumstances. 


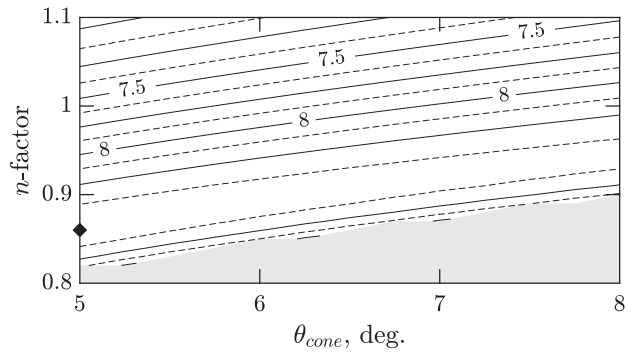

(a) Thrust $F_{z}$

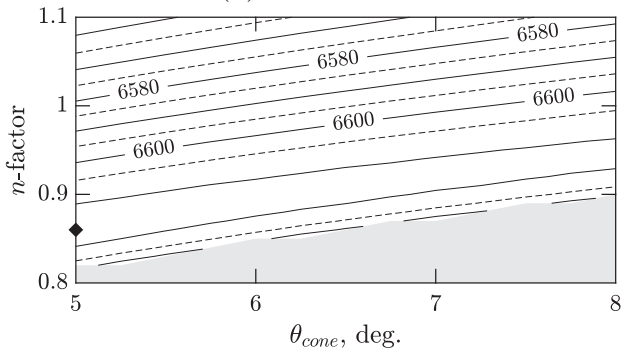

(c) Fuel-based specific impulse $I_{s p}$

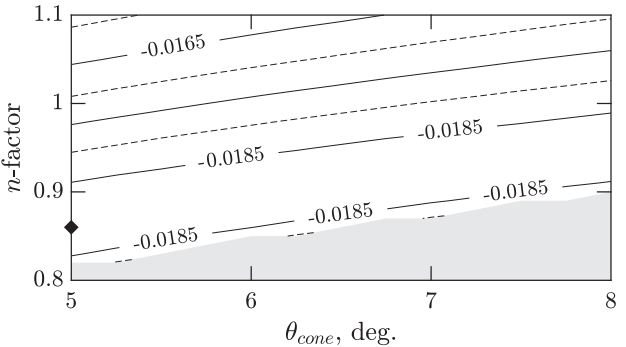

(b) Torque $\mathcal{T}$

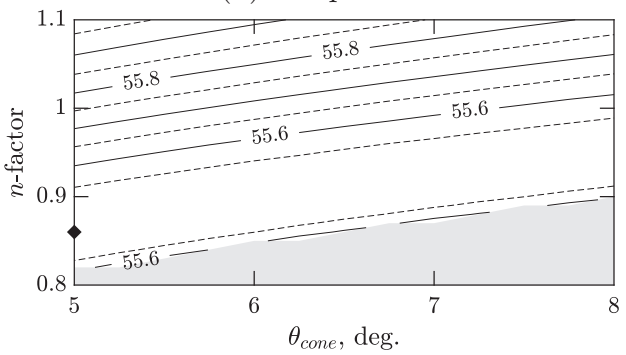

(d) TSFC, $\mathrm{kg} /(\mathrm{kN} \cdot \mathrm{h})$

Figure 9: Integrated RDE performance map, Mach 3, $\bar{q}=1000$ psf (48 kPa), hydrogen

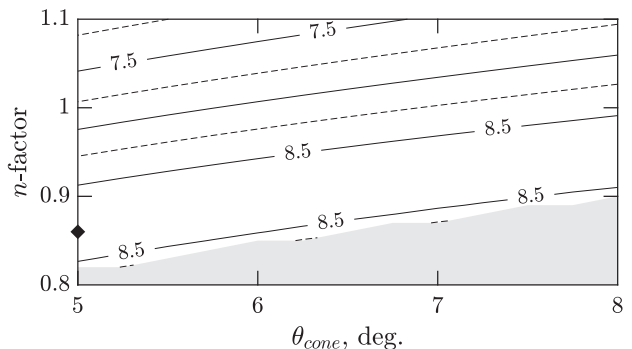

(a) Thrust $F_{z}, \mathrm{kN}$

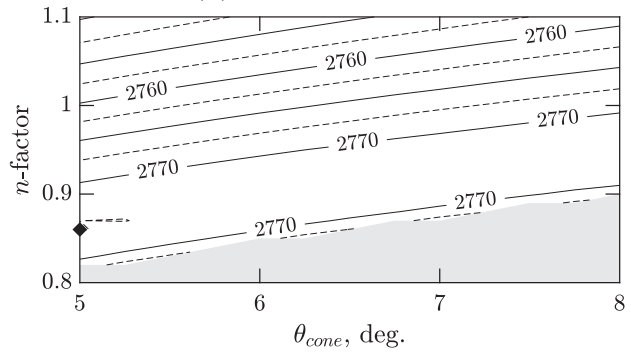

(c) Fuel-based specific impulse $I_{s p}, \mathrm{~s}$

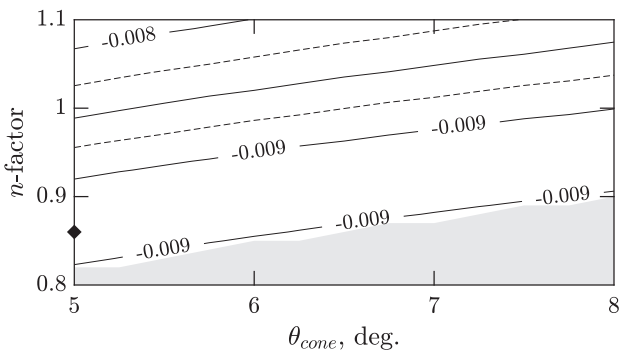

(b) Torque $\mathcal{T}, \mathrm{kN} \cdot \mathrm{m}$

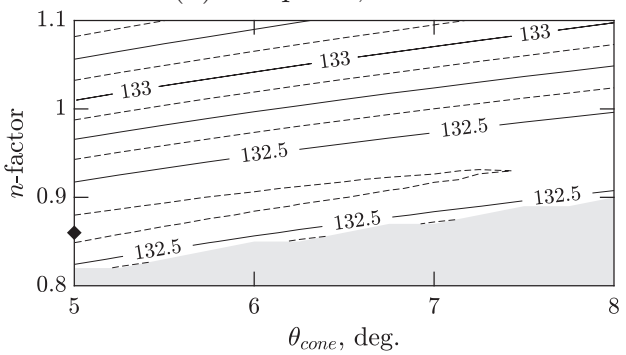

(d) TSFC, $\mathrm{kg} /(\mathrm{kN} \cdot \mathrm{h})$

Figure 10: Integrated RDE performance map, Mach 3, $\bar{q}=1000$ psf (48 kPa), propane 


\section{Volumetric Efficiency}

Figures 9 and 10 show peak performance across a swath of waverider forebody cone angle and $n$-factor combinations. To further evaluate the overall forebody/engine integration, the forebody's volumetric efficiency

$$
\eta_{v}=\frac{v^{2 / 3}}{S_{w}}
$$

can be considered, where $v$ is the forebody volume and $S_{w}$ is the wetted surface area [45]. In high-speed configurations, the volumetric efficiency is a suitable figure of merit as the wetted surface area relates to the convective heating to the vehicle. It has been long used for evaluating waverider configurations where obviously a large volume with a small wetted area is particularly attractive [46]. Since the installed RDE nozzle geometry and aft close-out region of the vehicles are not defined, the relative performance contributions from these features are removed from consideration. Consequently, for waverider forebody performance contribution, $v$ and $S_{w}$ are defined as the volume and wetted area of the forebody up to the start of the RDE. Additionally, a base area is not included in the wetted area calculations but which will not affect the overall conclusions.

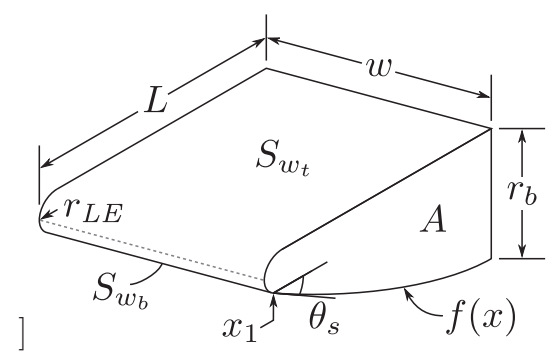

Figure 11: Schematic of forebody parameters for volumetric efficiency calculations

The volumetric efficiency is evaluated for a two-dimensional, blunt wedge shown schematically in Fig. 11 whose volume can be expressed as

$$
v=A w
$$

The area $A$ is the sum of two smaller areas which enclose a region at the leading edge and enclose the remaining forebody side view area

$$
A=\pi r_{L E}^{2}\left(\frac{\frac{\pi}{2}-\theta_{s}}{2 \pi}\right)+\int_{x_{1}}^{L} f(x) d x
$$

where $r_{L E}$ is the radius of the leading edge and $f(x)$ is the function representing the forebody geometry from Eq. (1). The integration extends over the region from $x_{1}$ (defined as the the interface point between the leading-edge hemicylinder and the lower surface specified by $f(x)$ ) to the trailing edge of the waverider $L$. The value of $x_{1}$ is determined by equating the two expressions for the leading-edge radius at this interface point

$$
\begin{aligned}
r\left(x_{1}\right) & =r_{L E} \cos \theta_{s} \\
& =r_{b}\left(\frac{x_{1}}{L}\right)^{n}
\end{aligned}
$$


yielding

$$
x_{1}=L\left(\frac{r_{L E} \cos \theta_{s}}{r_{b}}\right)^{1 / n}
$$

Since $\theta_{s}$ is also a function of $x_{1}$, this value is determined by bisection.

The wetted surface area of the blunt wedge is the sum of wetted areas from the top, bottom, and twice the area in the side view

$$
\begin{aligned}
S_{w} & =S_{w_{t}}+S_{w_{b}}+2 A \\
& =w\left[L-x_{1}+r_{L E}\left(1-\sin \theta_{s}\right)\right]+w\left[r_{L E}\left(\frac{\pi}{2}-\theta_{s}\right)+\int_{x_{1}}^{L} \sqrt{1+\left[f^{\prime}(x)\right]^{2}} d x\right]+2 A
\end{aligned}
$$

Recalling that a finite leading edge has been added to the forebody, the arc length for this region is that for a portion of the circumference of a circle over the angle $\left(\pi / 2-\cos \theta_{s}\right)$, plus the arc length using Eq. (1) beginning at $x_{1}$.

From data displayed in Figs. 9(d) and 10(d) for hydrogen and propane respectively, it was found that the volumetric efficiencies for either fuels are practically indistinguishable for each peak performing $\theta_{\text {cone }} / n$ combination. This is shown in Table 4 . For this range of cone angles, the data show a a 30 percent increase from the lowest to the higher value of volumetric efficiency for both hydrogen and propane fuels. Ffor a given level of TSFC performance, increased volumetric efficiency is driven by two distinct trends, increased cone angles and blunter shapes.

Table 4: Volumetric efficiencies for hydrogen and propane

\begin{tabular}{ccc}
\hline Cone Angle (deg.) & Peak $n$ & $\eta_{v}$ \\
\hline 5 & 0.86 & 0.0623 \\
6 & 0.89 & 0.0690 \\
7 & 0.92 & 0.0751 \\
8 & 0.95 & 0.0807 \\
\hline
\end{tabular}

\section{Range Impacts}

The relative effect of the lift-to-drag ratio arising from different cone angles and $n$ factors, and the propulsion efficiency of the RDE on the range can be explored through a modified form of the Breguet range equation. The Breguet range equation is given by

$$
R=I_{s p} V \frac{L}{D} \ln \left(\frac{m_{i}}{m_{f}}\right)
$$

where $R$ is the range, $I_{s p}$ is the specific impulse of the propulsion system, $V$ is the velocity, $L / D$ is the vehicular lift-to-drag ratio, and $m_{i}$ and $m_{f}$ are the vehicle masses at the start and end of cruise [45]. For the present study, instead of the entire vehicle, only the forebody is considered. For comparison purposes, a normalized range metric is defined by modifying the Breguet range equation, yielding

$$
\hat{R} \equiv I_{s p} \frac{L}{D} /\left(I_{s p} \frac{L}{D}\right)_{r e f}
$$




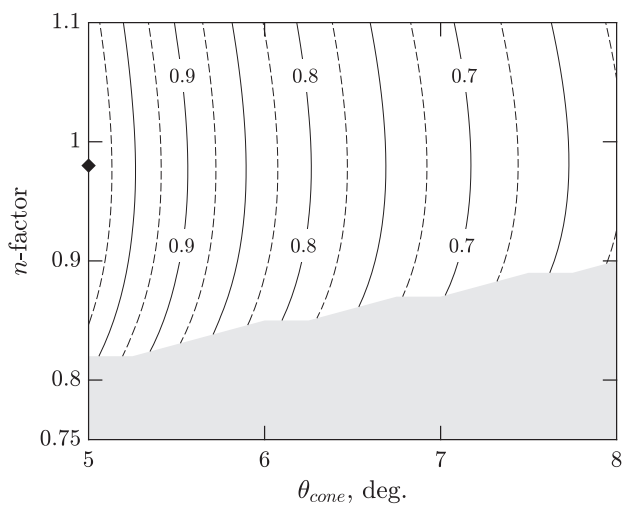

Figure 12: Normalized range metric; Mach 3, $\bar{q}=1000$ psf (48 kPa), hydrogen

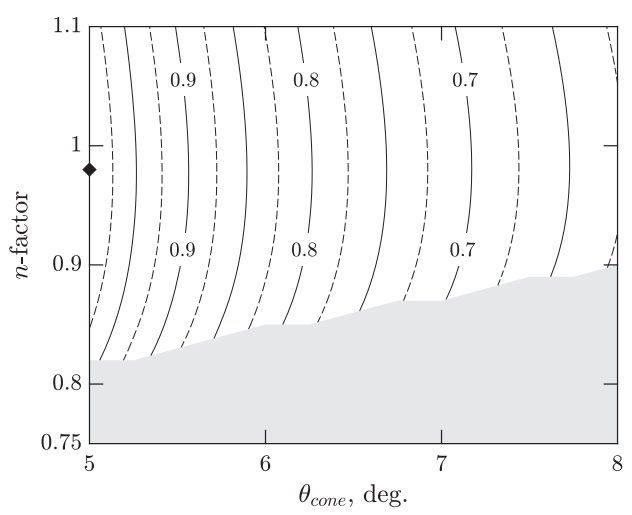

Figure 13: Normalized range metric; Mach 3, $\bar{q}=1000$ psf (48 $\mathrm{kPa})$, propane

where the ref condition is the greatest value of $I_{s p}(L / D)$ at $M_{\infty}=3$ and $\bar{q}=1000$ psf $(48 \mathrm{kPa})$ for the particular fuel.

Figure 12 shows the range metric $\hat{R}$ as a function of cone angle and $n$-factor at $M_{\infty}=3$ and $\bar{q}=1000 \mathrm{psf}\left(48 \mathrm{kPa}\right.$ ) for $\mathrm{H}_{2} /$ air. For these conditions, the best normalized range metric occurs at $\theta_{\text {cone }}=5 \mathrm{deg}$, and $n=0.98$. The figure shows a large region of relatively flat range metric, decreasing slowly with increasing cone angle. The better performance of small cone angles is due to the higher invisicid lift-to-drag ratios. Similar results are presented for propane, normalized by the greatest value for this particular fuel, in Fig. 13 and the plot looks similar to that of hydrogen. Being normalized, the actual range for a nominal vehicle is unavailable and is the subject of future work. Plots for dynamic pressures of $\bar{q}=500 \mathrm{psf}(24 \mathrm{kPa})$ and $\bar{q}=1500 \mathrm{psf}(72 \mathrm{kPa})$ are omitted as the contours differ negligibly from those presented.

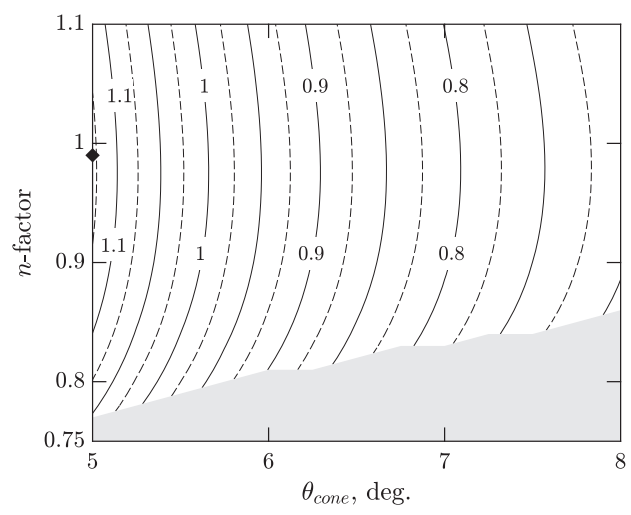

Figure 14: Normalized range metric; Mach $3.5, \bar{q}=1000 \operatorname{psf}(48 \mathrm{kPa})$, hydrogen

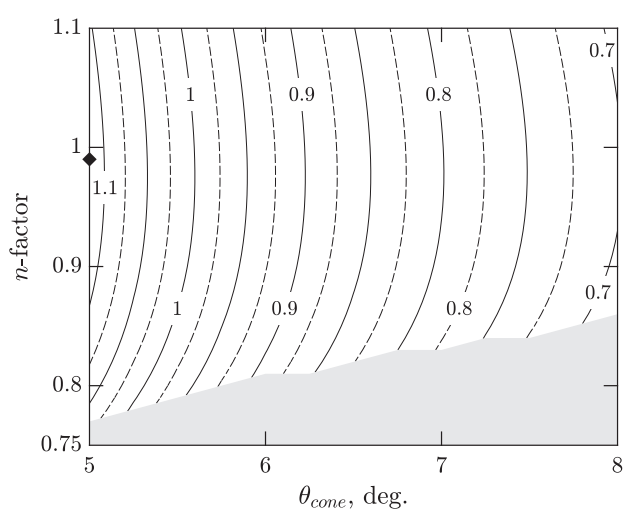

Figure 15: Normalized range metric; Mach $3.5, \bar{q}=1000 \operatorname{psf}(48 \mathrm{kPa})$, propane

Figures 14 and 15 show the normalized range metric data for Mach 3.5, and $\bar{q}=1000$ psf $(48 \mathrm{kPa})$ for hydrogen and propane respectively. Due to the normalization by the peak value at $M_{\infty}=3$ and $\bar{q}=1000$ psf for the respective fuels, values larger than unity are possible. The peak value of $\hat{R}$ at $M_{\infty}=3.5$ is 1.13 for hydrogen and 1.18 for propane, 
indicating 13 and 18 percent increases over $M_{\infty}=3$ respectively. The peaks occur at $\theta_{\text {cone }}=5 \operatorname{deg}$ and $n=0.97$.

\section{Limitations of the Analysis}

The results shown above indicate that the best performance is obtained regardless of whether the fuel is hydrogen or propane for the range of Mach numbers considered. It therefore appears that the OFWR is a robust design when integrated with an RDE. Note that the method assumes that the three-dimensional shock wave shape of the inviscid flowfield can be obtained by incorporating a series of known flowfields orientated on planes normal to the user defined shock wave trace in the base plane of the flowfield [46]. Further refinement would include the boundary layer.

Recently, Frolov et al. [22] have reported successful operation of a hydrogen-fueled RDE-powered ramjet in a Mach 5.7 freestream with flow stagnation temperatures of 1500 K. This operation presents flow conditions that exceed the assumptions in the present analysis, in particular the autoignition condition. A further understanding is required to properly extend the present study to higher Mach numbers.

Further, the present analysis does not model the RDE inlet. Flow into the RDE is approximated simply as a function of the forebody's exit stagnation pressure and not the inlet Mach number. Since thrust and mass flow rate both scale strongly with this parameter, this is potentially the cause of the insensitivity of specific impulse and TSFC to freestream and body shape parameters. Furthermore, each configuration is considered as "on-design," with the inlet size dictated by the RDE model. The inlet adapts to each successive design point. A detailed discussion of the limitations of the RDE model is given in [12].

\section{Conclusions}

High-speed, waverider forebodies constructed using the osculating flowfield possess characteristics favorable for the integration of ramjet and scramjet air-breathing propulsion systems. The present study replaced those systems with an RDE. Best performance is achieved for forebodies with small cone angles and convex power-law body geometries. Thrust and resultant torque are sensitive to body shape and freestream parameters, but not specific impulse and thrust-specific fuel consumption. Higher Mach numbers yielded improved propulsive performance but are limited by autoignition.

\section{References}

[1] K. Kailasanath, AIAA J. 38, 1698 (2000)

[2] D.T. Pratt et al., J. Propul. Power 7, 837 (1991)

[3] F.K. Lu, E.M. Braun, J. Propul. Power 30, 1125 (2014)

[4] K. Kailasanath, AIAA Paper 2017-0784 (2017)

[5] K. Kailasanath, AIAA J. 41, 145 (2003)

[6] G.D. Roy et al., Prog. Energy Combust. Sci. 30, 545 (2004)

[7] R.J. Pegg et al., AIAA Paper 1996-2918 (1996)

[8] D.R. Wilson et al., AIAA Paper 2001-1784 (2001)

[9] W.H. Heiser, D.T. Pratt, J. Propul. Power 18, 68 (2002) 
[10] E.M. Braun et al. Aerosp. Sci. Tech. 27, 201 (2013)

[11] R.T. Fievisohn, K.H. Yu, J. Propul. Power 33, 89 (2017)

[12] A.R. Mizener, F.K. Lu, J. Propul. Power 33, 1543 (2017)

[13] https://www.nationalmuseum.af.mil/Visit/Museum-Exhibits/FactSheets/Display/Article/195765/scaled-composites-long-ez-borealis/ cited 23 May 2019

[14] J. Kasahara et al., J. Propul. Power 25, 173 (2009)

[15] K. Goto et al., AIAA Paper 2018-0157 (2018)

[16] A.R. Mizener et al., J. Propul. Power 35, 289 (2019)

[17] A. Tobita et al., U.S. Patent 7,784,267 (2010)

[18] P. Wolański, Proc. Combustion Inst. 34, 125 (2013)

[19] R. Zhou, Chin. J. Aeron. 29, 15 (2015)

[20] B.A. Rankin et al., J. Propul. Power 33, 131 (2017)

[21] S.M. Frolov et al., Dokl. Phys. Chem 474, 75 (2017)

[22] S.M. Frolov et al., Dokl. Phys. Chem 481, 100 (2018)

[23] S. Liu et al., AIAA Paper 2017-2282 (2017)

[24] D. Sziroczak, H. Smith, Prog. Aerosp. Sci. 84, 1 (2016)

[25] P.E. Rodi, AIAA Paper 2005-0511 (2005)

[26] P.E. Rodi, AIAA Paper 2012-3223 (2012)

[27] K.D. Jones, K.B. Center, AIAA Paper 2002-3204 (2002)

[28] P.E. Rodi, D. Genovesi, AIAA Paper 2007-4344 (2007)

[29] M.A. Neer, AIAA J. 13, 924 (1975)

[30] R.S. Conti, M. Hertzberg, J. Fire Sci. 6, 348 (1988)

[31] R. Bounaceur et al., J. Engg. Gas Turbines Power 138, 021505 (2016)

[32] Anon., Engines, Aircraft, Turbojet and Turbofan, General Specification For (1973)

[33] D.G. Goodwin et al., Cantera: An Object-Oriented Software Toolkit for Chemical Kinetics, Thermodynamics, and Transport Processes, accessed 31 May 2017 (2017)

[34] Shock \& Detonation Toolbox, Caltech, accessed 15 March 2016 (2014)

[35] H. Wang et al., USC Mech Version II: High-Temperature Combustion Reaction Model of $\mathrm{H}_{2} / \mathrm{CO} / \mathrm{C}_{1}-\mathrm{C}_{4}$ Compounds, accessed 31 May 2017 (2007)

[36] C.A. Nordeen et al., Combustion, Explosion, and Shock Waves 50, 568 (2014)

[37] M. Sichel, J.C. Foster, Acta Astron. 6, 243 (1979)

[38] D.E. Paxson, AIAA Paper 2014-0284 (2014)

[39] C.A. Nordeen, D. Schwer, AIAA Paper 2015-3781 (2015)

[40] G.I. Maikapar, Fluid Dyn. 28, 696 (1993)

[41] F.A. Bykovskii et al., J. Propul. Power 22,1204 (2006)

[42] S.S. Grossel, Overview of Combustion and Flame Propagation Phenomena Related to DDAs, in Deflagration and Detonation Flame Arresters, (Wiley, Hoboken, New Jersey, 2002), pp. 51-75

[43] M. Kaneshige, J.E. Shepherd, Detonation Database, GALCIT Tech. Rep. FM97-8, 1997

[44] P.A. Diakow, Detonation Characteristics of Dimethyl Ether, Methanol and Ethanol Air Mixtures, Masters Thesis, Queen's University, Ontario, Canada, 2012

[45] J.J. Bertin, Hypersonic Aerothermodynamics, (AIAA, New York, 1993)

[46] F. Ding et al., Acta Astron. 140, 190 (2017) 